\title{
A General Class of Convexification Transformation for the Noninferior Frontier of a Multiobjective Program
}

\author{
Tao Li ${ }^{1}$, Yanjun Wang ${ }^{2}$, Zhian Liang ${ }^{2}$ \\ ${ }^{1}$ Department of Statistics, Missouri University of Science \& Technology, Rolla, USA \\ ${ }^{2}$ Department of Mathematics Shanghai University of Finance and Economics, Shanghai, China \\ Email: tl6gc@mail.mst.edu
}

Received March 7, 2012; revised April 17, 2012; accepted May 2, 2012

Copyright (C) 2013 Tao Li et al. This is an open access article distributed under the Creative Commons Attribution License, which permits unrestricted use, distribution, and reproduction in any medium, provided the original work is properly cited.

\begin{abstract}
A general class of convexification transformations is proposed to convexify the noninferior frontier of a multiobjective program. We prove that under certain assumptions the noninferior frontier could be convexified completely or partly after transformation and then weighting method can be applied to identify the noninferior solutions. Numerical experiments are given to vindicate our results.
\end{abstract}

Keywords: Noninferior Frontier; Convexification; Weighting Method; Multiobjective Optimization

\section{Introduction}

In this paper, we consider the following multiobjective optimization problem:

$$
(P):\left\{\begin{array}{l}
\min f(x)=\left[f_{1}(x), f_{2}(x), \cdots, f_{k}(x)\right] \\
\text { s.t. } g_{j}(x) \leq 0, \quad j=1,2, \cdots, m,
\end{array}\right.
$$

where $k>1$ and $x \in R^{n}$ is a decision vector, $f_{i} \in C^{2}$, $i=1,2, \cdots, k$, are objective functions and $g_{j} \in C^{2}$, $j=1,2, \cdots, m$, are constraint functions.

Let $X=\left\{x \mid g_{j}(x) \leq 0, j=1,2, \cdots, m.\right\}$ be the feasible region in the decision space and

$$
F=\left\{\left[f_{1}(x), f_{2}(x), \cdots, f_{k}(x)\right] \mid x \in X\right\}
$$

be the feasible region in the objective space. A solution $x^{*}$ to Problem $(\mathrm{P})$ is called noniferior solution if there is no other feasible solution $x$ such that $f_{i}(x) \leq f_{i}\left(x^{*}\right)$, $i=1,2, \cdots, k$, with at least one strict inequality.

Let $X^{*}=\{x \mid x \in X$ and is noninferior solution $\}$ be the set of all the noninferior solutions in the decision space and

$$
F^{*}=\left\{\left[f_{1}(x), f_{2}(x), \cdots, f_{k}(x)\right] \mid x \in X^{*}\right\}
$$

be the set of noninferior points in the objective space and $F^{*}$ is also called the noninferior frontier of Problem (P).

An important problem in multiobjective optimization is to find the set of noninferior solutions. Many methods that are intended to identify noninferior solutions have been proposed such as the weighting method, weighting $p$-norm method, the $\infty$-norm method and the $\xi$-constraint method. Among these methods the weighting method is one of the simplest methods. In fact, the weighting method transforms multiple objectives into the following weighted sum by introducing weighting vector $\left(w_{1}, \cdots w_{k}\right)$ :

$$
(S P):\left\{\begin{array}{l}
\min \sum_{i=1}^{k} w_{i} f_{i}(x) \\
\text { s.t. } g_{j}(x) \leq 0, j=1,2, \cdots, m,
\end{array}\right.
$$

where

$\left(w_{1}, \cdots, w_{k}\right) \in W=\left[\left(w_{1}, \cdots, w_{k}\right) \mid \sum_{i=1}^{k} w_{i}=1\right.$ and $\left.w_{i} \geq 0\right]$. It is well-known that the optimal solution of Problem (SP) is the noninferior solution of Problem (P). Let $w^{*}$ be an optimal solution of Problem (SP) with $w=w^{*}$, then we have

$$
\sum_{i=1}^{k} w_{i}^{*} f_{i}(x) \geq \sum_{i=1}^{k} w_{i}^{*} f_{i}\left(x^{*}\right) \forall x \in X .
$$

By the definition of supporting hyperplane we know that there exists a supporting hyperplane of $F^{*}$ at $\left(f_{1}\left(x^{*}\right), \cdots, f_{k}\left(x^{*}\right)\right)$, which is $\sum_{i=1}^{k} w_{i}^{*} y_{i}$. Thus the existence of a supporting hyperplane at the noninferior solution in the $\left[\left(f_{1}(x), \cdots, f_{k}(x)\right)\right]$-space which separates all the noninferior points one side is a necessary condition to guarantee the successful finding of noninferior solutions of Problem (P) by using weighting method. However, in many nonconvex circumstances, supporting 
hyperplane does not exist at some points of the noninferior frontier. Therefore, weighting method always fails to identify all the noninferior solutions in these cases.

Recently, convexification method has been successfully adopted in many subjects of optimization. For example, in [1-3] a series of convexification methods are proposed to process some classes of global optimization problems with certain monotone properties and in [4,5] convexification schemes are presented to convexify the perturbation function and Lagrangian function in the dual search methods for nonlinear programming. In [6,7], a general convexification and concavification scheme are proposed for certain classes of monotone and nonmonotone optimization problems. The scheme converts the problems into classes of concave and reverse convex programming problems with better structures. Li et al derived a general convexification method for nonconvex minimization problems in [8]. Their method transforms the problems into convex ones and thus the local techniques can be used to solve the new problems. A reciprocal transformation for the convexification of posynomial programs with positive variables are presented in [9]. In [10-12], $p$-power and exponential generating method were used as a special convexification transformations and they proved that under certain assumptions, by applying the $p$-power or exponential generating method to objective function, the noninferior frontier of a multiobjective problem can be convexified completely or partly and then the weighting method can be applied to identify the noninferior solutions. However, due to the various forms of objective functions, $p$-power might not always serve as an efficient transformation. Thus the choice range of such transformations should be enlarged.

The main purpose of this paper is to present a class of general convexification transformation methods to convexify the noninferior frontier of a multiobjective problem. Compared with previous works, the major contributions of our paper are as follows:

- We prove that the noninferior frontier could be convexified completely or partially by applying a more general transformation under certain assumptions. Also, we generalized the results in [10].

- Our transformation further expands the class of multiobjective program that weighting method could solve by designing the transformation function based on the objective function. Our transformation can handle practical problems more efficiently than the one in [10] as well.

The paper is organized as follows: in Sections 2 and 3, a general form of transformation is proposed and then we prove that under some assumptions the noninferior frontier could be convexified completely or partly. In Section 4 some examples are given to vindicate our results. We give a conclusion about this paper in the last section.

\section{Convexification of Noninferior Frontier}

As in [10], the noninferior frontier of Problem (P) can be expressed as

$$
f_{k}=\psi\left(f_{1}, f_{2}, \cdots, f_{k-1}\right),
$$

where $\psi$ is a nonincreasing function of $f_{i}, i=1,2, \cdots, k-1$ at $F^{*}$.

Consider the following transformation of the objective functions

$$
f_{i p}=h_{p}\left(f_{i}\right), i=1,2, \cdots, k,
$$

where $h_{p}: Z_{p} \rightarrow R$ is strictly increasing where $p>0$ is a parameter, and $f_{i}\left(X^{*}\right) \subseteq Z_{p} \subseteq R, i=1,2, \cdots, k$. Then Problem $(\mathrm{P})$ is transformed to a new problem which reads

$$
(C P):\left\{\begin{aligned}
& \min f(x)= {\left[h_{p}\left(f_{1}(x)\right), h_{p}\left(f_{2}(x)\right), \cdots,\right.} \\
&\left.h_{p}\left(f_{k}(x)\right)\right] \\
& \text { s.t. } \quad g_{j}(x) \leq 0, \quad j=1,2, \cdots, m .
\end{aligned}\right.
$$

Let $X_{c p}^{*}$ be the sets of all the noninferior solutions for Problem (CP), then $X^{*}=X_{c p}^{*}$.

Proof. For any point $x^{*} \in X^{*}$, if $x^{*} \notin X_{c p}^{*}$, then $\exists x_{c p}^{o} \in X$ such that

$h_{p}\left(f_{i}\left(x_{c p}^{o}\right)\right) \leq h_{p}\left(f_{i}\left(x^{*}\right)\right), i=1,2, \cdots, k$, with at least one strict inequality. Without loss of generality, we assume $h_{p}\left(f_{1}\left(x_{c p}^{o}\right)\right) \leq h_{p}\left(f_{1}\left(x^{*}\right)\right)$. Since $h_{p}(x)$ is strictly increasing, we must have $f_{i}\left(x_{c p}^{o}\right) \leq f_{i}\left(x^{*}\right), i=1,2, \cdots, k$, with strict inequality holds at $i=1$. Then $x^{*}$ is not a noninferior point which contradicts the assumption. So, $x^{*} \in X_{c p}^{*}$. Therefore, $X^{*} \subseteq X_{c p}^{*}$. Similarly, it can be shown that $X_{c p}^{*} \subseteq X^{*}$.

The noninferior frontier of Problem (CP) can be expressed as

$$
\begin{aligned}
& h_{p}\left(f_{k}(x)\right) \\
= & \bar{\psi}\left(h_{p}\left(f_{1}(x)\right), \cdots, h_{p}\left(f_{k}(x)\right)\right) \\
= & h_{p}\left[\psi\left(h_{p}^{-1}\left(h_{p}\left(f_{1}(x)\right)\right), \cdots, h_{p}^{-1}\left(h_{p}\left(f_{k-1}(x)\right)\right)\right)\right] .
\end{aligned}
$$

Let $H(x)$ be the Hessian matrix of $\psi(x)$ and $\bar{\lambda}=\inf _{x \in X^{*}} \lambda_{0}(x)$, where $\lambda_{0}(x)$ is the minimum eigenvalue of $H(x)$. Further, we make the following assumptions:

I) $\psi$ is a twice continuous differentiable function and $\frac{\partial \psi}{\partial f_{i}}$ is negative for all $x \in X^{*}$, and $\frac{\partial f_{k}}{\partial f_{i}}<-\eta_{0}$, $\forall x \in X^{*}$ and $i=1,2, \cdots, k-1$, where $\eta_{0}$ is positive. Moreover, $\bar{\lambda}>-\infty$

II) $h_{p}: Z_{p} \rightarrow R$ is twice differentiable and $h_{p}^{\prime \prime}\left(f_{i}(x)\right) \rightarrow+\infty, \forall x \in X^{*}$, as $p \rightarrow+\infty, i=1,2, \cdots, k-1$. 


$$
h_{p}^{\prime \prime}\left(f_{k}(x)\right)>-M, \forall x \in X^{*}, p>0,
$$

where $M$ is a positive number.

III) $X^{*}$ is a compact set.

Then we have the following theorem: Suppose that assumptions I)-III) are satisfied. Then there exists a $p_{0}>0$ such that when $p>p_{0}$ the noninferior frontier of Problem (CP) is convex.

Proof. Let $y_{i}=f_{i}(x), \bar{y}_{i}=h_{p}\left(f_{i}(x)\right), i=1,2, \cdots, k$ -1 , and $H_{p}(x)$ denote the Hessian matrix of $\bar{\psi}(x)$.

Then we have

$$
\begin{gathered}
h_{p}\left(f_{k}\right)=h_{p}\left[\psi\left(h_{p}^{-1}\left(\bar{y}_{1}\right), \cdots, h_{p}^{-1}\left(\bar{y}_{k-1}\right)\right)\right] . \\
\frac{\partial h_{p}\left(f_{k}\right)}{\partial \bar{y}_{i}}=h_{p}^{\prime}\left(f_{k}\right) \frac{\partial f_{k}}{\partial f_{i}} \frac{1}{h_{p}^{\prime}\left(y_{i}\right)}<0, i=1, \cdots, k-1 . \\
\frac{\partial^{2} h_{p}\left(f_{k}\right)}{\partial \bar{y}_{i}^{2}} \\
=h_{p}^{\prime \prime}\left(f_{k}\right)\left(\frac{\partial f_{k}}{\partial f_{i}}\right)^{2}\left(\frac{1}{h_{p}^{\prime}\left(y_{i}\right)}\right)^{2}+h_{p}^{\prime}\left(f_{k}\right) \frac{\partial^{2} f_{k}}{\partial f_{i}^{2}}\left(\frac{1}{h_{p}^{\prime}\left(y_{i}\right)}\right)^{2} \\
+h_{p}^{\prime}\left(f_{k}\right) \frac{\partial f_{k}}{\partial f_{i}} \frac{h_{p}^{\prime \prime}\left(y_{i}\right)}{\left(h_{p}^{\prime}\left(y_{i}\right)\right)^{2}} .
\end{gathered}
$$

when $i \neq j$

$$
\begin{aligned}
\frac{\partial^{2} h_{p}\left(f_{k}\right)}{\partial \bar{y}_{i} \partial \bar{y}_{j}}= & h_{p}^{\prime \prime}\left(f_{k}\right) \frac{\partial f_{k}}{\partial f_{i}} \frac{\partial f_{k}}{\partial f_{j}} \frac{1}{h_{p}^{\prime}\left(y_{i}\right)} \frac{1}{h_{p}^{\prime}\left(y_{j}\right)} \\
& +h_{p}^{\prime}\left(f_{k}\right) \frac{\partial^{2} f_{k}}{\partial f_{i} \partial f_{j}} \frac{1}{h_{p}^{\prime}\left(y_{i}\right)} \frac{1}{h_{p}^{\prime}\left(y_{j}\right)}
\end{aligned}
$$

Let

$$
\begin{gathered}
\alpha=\left(\frac{\partial f_{k}}{\partial f_{1}}, \cdots, \frac{\partial f_{k}}{\partial f_{k-1}}\right)^{\mathrm{T}}, \\
A=\operatorname{diag}\left(\frac{1}{h_{p}^{\prime}\left(y_{1}\right)}, \cdots, \frac{1}{h_{p}^{\prime}\left(y_{k-1}\right)}\right), \\
B=\operatorname{diag}\left(\frac{-\partial f_{k}}{\partial f_{1}} h_{p}^{\prime \prime}\left(y_{1}\right), \cdots, \frac{-\partial f_{k}}{\partial f_{k-1}} h_{p}^{\prime \prime}\left(y_{k-1}\right)\right) .
\end{gathered}
$$

Then

$$
H_{p}(x)=A\left(h_{p}^{\prime \prime}\left(f_{k}\right) \alpha \alpha^{\mathrm{T}}+H(x)+B(x)\right) A .
$$

From (2.1) and (2.2), we have that $H_{p}(x)$ is a positive definite matrix if and only if

$\left(h_{p}^{\prime \prime}\left(f_{k}\right) \alpha \alpha^{\mathrm{T}}+H(x)+B(x)\right)$ is a positive definite matrix.

We assume that $\bar{\lambda}<0$, otherwise $H(x)$ is already positive definite.

Further, let

$$
N=\max _{d \in S_{n}, x \in X^{*}} d^{\mathrm{T}} \alpha<+\infty .
$$

where $S_{n}$ denotes the unit sphere in $R^{n}$.

By II) and III), we have

$$
h_{p}^{\prime \prime}\left(y_{i}\right) \Rightarrow+\infty, \text { as } p \rightarrow+\infty, \forall x \in X^{*}, i=1,2, \cdots, k-1 \text {. }
$$

Therefore, for $\frac{M N-\bar{\lambda}}{\eta_{0}}$, there exists $p_{0}>0$ such that for any $p>p_{0}$, we have

$$
h_{p}^{\prime \prime}\left(y_{i}\right)>\frac{M N-\bar{\lambda}}{\eta_{0}}, \forall x \in X^{*}, i=1,2, \cdots, k-1 .
$$

Then $\forall d \in S_{n}$, we have

$$
\begin{aligned}
& d^{\mathrm{T}}\left(h_{p}^{\prime \prime}\left(f_{k}\right) \alpha \alpha^{\mathrm{T}}+H(x)+B(x)\right) d \\
= & h_{p}^{\prime \prime}\left(f_{k}\right)\left(d^{\mathrm{T}} \alpha\right)^{2}+d^{\mathrm{T}} H(x) d+d^{\mathrm{T}} B(x) d \\
= & h_{p}^{\prime \prime}\left(f_{k}\right)\left(d^{\mathrm{T}} \alpha\right)^{2}+d^{\mathrm{T}} H(x) d+\sum_{i=1}^{k-1} \frac{-\partial f_{k}}{\partial f_{i}} h_{p}^{\prime \prime}\left(y_{i}\right) d_{i}^{2} \\
\geq & -M N+\bar{\lambda}+\sum_{i=1}^{k-1} \frac{-\partial f_{k}}{\partial f_{i}} h_{p}^{\prime \prime}\left(y_{i}\right) d_{i}^{2}>0 .
\end{aligned}
$$

Then $\left(h_{p}^{\prime \prime}\left(f_{k}\right) \alpha \alpha^{\mathrm{T}}+H(x)+B(x)\right)$ is a positive definite matrix when $p>p_{0}$. Therefore, the noninferior frontier of Problem (CP) is convex when $p>p_{0}$ and we complete the proof.

Corollary 2.2 Suppose that assumptions I)-III) are satisfied. Then there is a $p_{1}$ such that the supporting hyperplane exists everywhere on the noninferior frontier of Problem (CP) when $p>p_{1}$.

Proof. By theorem 2.2, there exists a $p_{1}$ such that the noninferior frontier is convex everywhere when $p>p_{1}$, and then by [13] we know that the supporting hyperplane exists everywhere.

Further by the discussion above, we can obtain the following corollary:

Corollary 2.2 Suppose that assumptions I)-III) hold. Then $x^{*}$ is a noninferior solution of Problem (P) if and only if there exists a $p_{1}$ such that $x^{*}$ is an optimal solution of Problem (CP) with $w=w_{p}^{*} \in W$ when $p>p_{1}$.

Remark 1. If we set $h_{p}(y)=y^{p}$, it is easy to verify that $h_{p}(y)$ satisfies assumption II). The primal problem could be transformed into the following problem:

$$
(C P): \begin{cases}\min & f(x)=\left[f_{1}^{p}(x), f_{2}^{p}(x), \cdots, f_{k}^{p}(x)\right] \\ \text { s.t. } & g_{j}(x) \leq 0, j=1, \cdots, m .\end{cases}
$$

Note that (2.3) is exactly the transformation proposed in [10].

Remark 2. We can derive other types of transformations by constructing many specific function forms satisfying assumption II). For example, each of functions 
$(y+l)^{p}, \quad \mathrm{e}^{(y+l)^{p}}, \quad \ln ^{p}(y+l), \ln \left(1+(l+y)^{p}\right)$, where $(l+y)>0$ and $\mathrm{e}^{p(y+l)}, \ln \left(1+\mathrm{e}^{p y}\right)$ could be used as $h_{p}(y)$.

\section{Local Convexification of the Noninferior Frontier}

In some cases, assumptions I)-III) may not hold simultaneously. For instance, $\psi(x)$ might not be twice continuous differentiable or $\bar{\lambda}=-\infty$. In these circumstances, it might be difficult to globally convexify the noninferior frontier, however, we can achieve the local convexification of the noninferior frontier. Assume that assumptions I)-III) hold in a compact neighborhood $N\left(x^{*}\right) \subseteq X^{*}$, then there exists a $p_{1}$ such that the Hessian matrix $H_{p}(x)$ of $\bar{\psi}(x)$ is positive definite for $x \in N\left(x^{*}\right)$ in the $\left\{h_{p}\left(f_{1}\right), \cdots, h_{p}\left(f_{k}\right)\right\}$-space when $p>p_{1}$.

Proof. Theorem 3.1 could be vindicated by the similar way used in the proof of Theorem 2.2.

Then similar to corollaries 2.1 and 2.2, we have the following corollaries:

Corollary 3.1 Suppose that assumptions I)-III) hold in a compact neighborhood $N\left(x^{*}\right) \subseteq X^{*}$, then there exists a $p_{1}$ such that supporting hyperplane exists on the confined noninferior frontier sector $\left\{h_{p}\left(f_{1}\right), \cdots, h_{p}\left(f_{k}\right) \mid x \in N\left(x^{*}\right)\right\}$ for all interior points of $N\left(x^{*}\right)$ when $p>p_{1}$.

Corollary 3.2 Assume that a noninferior solution $x^{*} \in X^{*}$ has a compact neighborhood $N\left(x^{*}\right) \subseteq X^{*}$ and assumptions I)-III) hold in $N\left(x^{*}\right)$, then for $p$ large enough there exists a $w_{p}^{*} \in W$ such that $x^{*}$ is a local optimal solution of Problem (P) with $w=w_{p}^{*}$.

\section{Numerical Experiments}

Example 1: Consider the following example:

$(1):\left\{\begin{array}{l}\min \left[f_{1}(x)=\mathrm{e}^{x_{1}}, f_{2}(x)=\mathrm{e}^{x_{2}}\right] \\ \text { s.t. } x_{2}-\sqrt{9-\frac{9}{4} x_{1}^{2}} \geq 0, \\ 0 \leq x_{1} \leq 1, \\ 1 \leq x_{2} \leq 2.99 .\end{array}\right.$

The noninferior frontier of Problem (1) is

$\psi(x)=f_{2}(x)=\mathrm{e}^{\sqrt{9-\frac{9}{4}\left(\ln f_{1}(x)\right)^{2}}}, 1.1772 \leq f_{1}(x) \leq 2.7183$.

Figure 1 depicts the feasible region of Problem (1). From Figure 1 we know that the noninferior frontier of Problem (1) is not convex, thus weighting method would not identify all the noninferior solutions in this case.

Let $h_{p}(y)=\ln ^{p}(x)$ and $p=2$, then the Problem (1) could be transformed to the following problem: $(2):\left\{\begin{array}{l}\min \left[f_{1}^{\prime}(x)=x_{1}^{2}, f_{2}^{\prime}(x)=x_{2}^{2}\right] \\ \text { s.t. } x_{2}-\sqrt{9-\frac{9}{4} x_{1}^{2}} \geq 0, \\ 0 \leq x_{1} \leq 1, \\ 1 \leq x_{2} \leq 2.99 .\end{array}\right.$

Then the noninferior frontier of Problem (2) is

$$
\bar{\psi}(x)=f_{2}^{\prime}(x)=9-\frac{9}{4} f_{1}^{\prime}, 0.0266 \leq f_{1}^{\prime}(x) \leq 1 .
$$

Figure 2 depicts the noninferior frontier of Problem (2). Clearly, the noninferior frontier of Problem (2) is convex and then we can identify all the noninferior solution of Problem (2) by applying the weighting method. Also, it's worthwhile to point out that compared to the transformation we used, the $p$-power transformation may produce a much more complicate problem.

Example 2: Consider the following example:

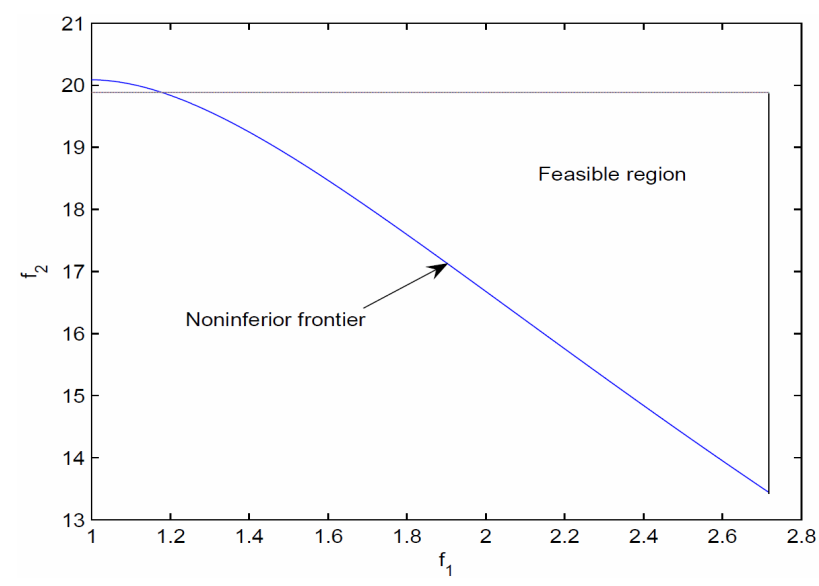

Figure 1. Feasible region and noninferior frontier of Problem (1).

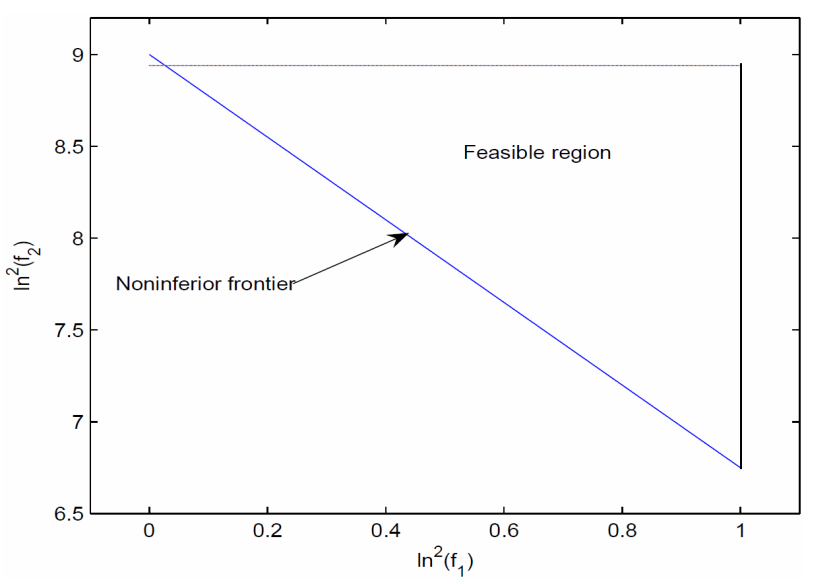

Figure 2. Feasible region and noninferior frontier of Problem (2). 
$(3):\left\{\begin{array}{l}\min \left[f_{1}(x)=-x_{1}^{2} x_{2}^{2}, f_{2}(x)=x_{1}+x_{2}\right], \\ \text { s.t. } \quad x_{1}, x_{2}>0 .\end{array}\right.$

Figure 3 depicts the feasible region of Problem (3). From Figure 3 we know that the noninferior frontier of Problem (3) is nonconvex. Note that the noninferior frontier of Problem (3) can be expressed as

$$
f_{2}(x)=\psi(x)=2\left(-f_{1}(x)\right)^{0.25} \text {. }
$$

And the hessian matrix of $\psi(x)$ is $-\frac{3}{8}\left(-f_{1}(x)\right)^{-1.75}$. It is evident that

$$
\lim _{x \rightarrow 0-}-\frac{3}{8}\left(-f_{1}(x)\right)^{-1.75}=-\infty,
$$

which contradicts assumption I). Thus we might not be able to completely convexify the noninferior frontier of Problem (3) by using our transformation method. However we could achieve the local convexification.

Let $h_{p}(y)=\mathrm{e}^{(p(x+1))}$ and $p=2$, then the Problem (3) could be transformed to the following problem:

(4): $\left\{\begin{array}{l}\min \left[f_{1}^{\prime}(x)=\mathrm{e}^{-2 x_{1}^{2} x_{2}^{2}+2}, f_{2}^{\prime}(x)=\mathrm{e}^{2 x_{1}+2 x_{2}+2}\right] \\ \text { s.t. } x_{1}, x_{2}>0 .\end{array}\right.$

Obviously, as shown in Figure 4, the noninferior frontier of Problem (4) is locally convex and then we can identify part of the noninferior solutions of Problem (4) by applying the weighting method.

\section{Conclusion}

As one of the simplest methods to identify the noninferior solutions of multiobjective problems, weighting method fails in many nonconvex cases. In this paper, a general class of convexification transformations is presented and we prove that the transformation could con-

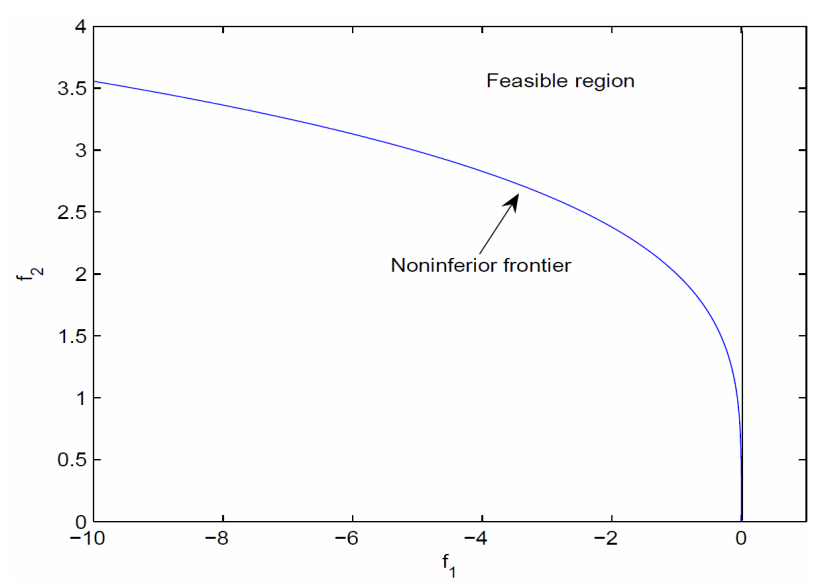

Figure 3. Feasible region and noninferior frontier of Problem (3).

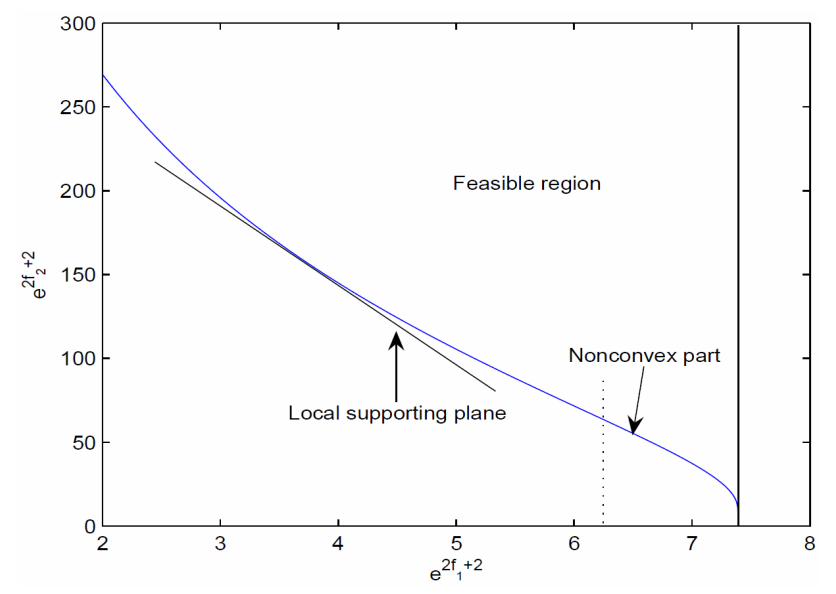

Figure 4. Feasible region and noninferior frontier of Problem (4).

vexify the noninferior frontier completely or partly under assumptions and then weighting method can be used successfully. This paper expands greatly the class of multiobjective programs that weighting method can cope with and provides more specific transformations to tackle practical problems efficiently.

\section{Acknowledgements}

The research was supported by National Natural Science Foundation of China under Project 10261005 and partially supported by National Natural Science Foundation of China under Project 10601030.

\section{REFERENCES}

[1] D. Li, X. L. Sun, M. P. Biswal and F. Gao, "Convexification, Concavification and Monotonization in Global Optimization," Annals of Operations Research, Vol. 105, No. 1-4, 2001, pp. 213-226. doi:10.1023/A:1013313901854

[2] X. L. Sun, K. McKinnon and D. Li, "A Convexification Method for a Class of Global Optimization Problem with Application to Reliability Optimization," Journal of Global Optimization, Vol. 21, No. 2, 2001, pp. 185-199. doi:10.1023/A:1011962605464

[3] Z. Y. Wu, F. S. Bai and L. S. Zhang, "Convexification and Concavification for a General Class of Global Optimization Problems," Journal of Global Optimization, Vol. 31, No. 1, 2005, pp. 45-60. doi:10.1007/s10898-004-0569-6

[4] D. Li, "Zero Duality Gap for a Class of Nonconvex Optimization Problems," Journal of Optimization Theory and Applications, Vol. 85, No. 2, 1995, pp. 309-324. doi:10.1007/BF02192229

[5] D. Li and X .L. Sun, "Local Convexification of Lagrangian Function in Nonconvex Optimization," Journal of Optimization Theory and Applications, Vol. 104, No. 1, 2000, pp. 109-120. doi:10.1023/A:1004628822745

[6] Z. Y. Wu, F. S. Bai and L. S. Zhang, "Convexification 
and Concavification for a General Class of Global Optimization Problems," Journal of Global Optimization, Vol. 31, No. 1, 2005, pp. 45-60. doi:10.1007/s10898-004-0569-6

[7] Z. Y. Wu, H. W. J. Lee and X. M. Yang, "A Class of Convexification and Concavification Methods for NonMonotone Optimization Problems," Optimization, Vol. 54 , No. 6, 2005, pp. 605-625. doi:10.1080/02331930500342807

[8] T. Li, Y. J. Wang, Z. Liang and P. M. Pardalos, "Local Saddle Point and a Class of Convexification Methods for Nonconvex Optimization Problems," Journal of Global Optimization, Vol. 38, No. 3, 2007, pp. 405-419. doi:10.1007/s10898-006-9090-4

[9] H. L. Li, J. F. Tsai and C. A. Floudas, "Convex Underestimation for Posynomial Functions of Positive Variables," Optimization Letter, Vol. 2, No. 3, 2008, pp. 333-340.

$$
\text { doi:10.1007/s11590-007-0061-6 }
$$

[10] D. Li, "Convexification of Noninferior Frontier," Journal of Optimization Theory and Applications, Vol. 88, No. 1, 1996, pp. 177-196. doi:10.1007/BF02192028

[11] C. J. Goh and X. Q. Yang, "Convxification of a Noninferior Frontier," Journal of Optimization Theory and Applications, Vol. 97, No. 3, 1998, pp. 759-768. doi:10.1023/A:1022654528902

[12] D. Li and M. P. Biswal, "Exponential Transformation in Convexifying a Noninferior Frontier and Exponential Generating Method," Journal of Optimization Theory and Applications, Vol. 99, No. 1, 1998, pp. 183-199. doi:10.1023/A:1021708412776

[13] M. S. Bazaraa, H. D. Sherali and C. M. Shetty, "Nonlinear Programming, Theory and Algorithms," 2nd Edition, John Wiley \& Sons, Inc., New York, 1993. 